\title{
New method for wind potential prediction using recurrent artificial neural networks
}

\author{
Yousra Amellas $^{1}$, Saif Serag ${ }^{1,3}$, Fahd Loukdache $^{1}$, Abdelouahed Djebli $^{2}$, , Adil Echchelh ${ }^{1}$ \\ ${ }^{1}$ Laboratory of Electronic treatment information, mechanic and energetic, Ibn Tofail University, Kenitra, Morocco. \\ ${ }^{2}$ Laboratory of Energetics, Fluid Mechanics and Materials, Abdelmalek Essaadi, Tetouan, Morocco. \\ ${ }^{3}$ Department of Energy Physics, Ministry of education, Yemen
}

\begin{abstract}
The aim of the study is to find the right architecture of the NARX neural network, in order to perform the daily prediction of the maximum wind speed of Laayoune city. We relied on the LevenbergMarquardt optimization algorithm. The RMSE error metric showed that NARX-SP outperforms NARX-P.
\end{abstract}

\section{Introduction}

The fight against climate change requires ecological solutions such as renewable energies [1-3]. We are interested in wind power [4], which considers itself an important source for the production of green electricity. Wind technology is totally dependent on wind, which is a very variable meteorological parameter. Its intermittence creates endless fluctuations in the production process, these variations directly influence the stability of the energy produced. The management of wind production is based on several stages, prediction at different time scales are essential.

Our study mainly focuses on short-term wind prediction [5]. Several methods can solve the wind prediction problem in a different way but we choose the statistical methods [6]. These statistical methods are also divided into several sub-methods. In the literature, we find statistical methods based on time series such as the best-known models ARMA (Auto-Regressive Moving Average) [7], ARIMA (Auto-Regressive Integrated Moving Average) is the most used model in the wind energy field which were established by box and Jenkins, ARX( Autoregressive with Exogenous Inputs)... which are linear models. While wind is characterized by a nonlinear behavior. For this reason, we propose the most recent methods which are the methods of artificial intelligence (AI) like ensemble methods, for example, random forests, decision trees, as well as other types of methods like SVR (Support Vector Regression) ....and also artificial neural network, of which there are two types of networks, those inspired by the electrical part of a biological neural network, and those inspired by its chemical part. The former are the case of our study, of which we have chosen from which we have chosen the NARX neuron network, in its two forms, NARX-SP (open loop) and NARX-P (closed loop).

The aim of our work is to compare NARX-P and NARX-SP in order to find the best model among them that will be able to predict the short-term maximum wind speed of Laayoune city. NARX-P and NARX-SP have been optimized by the Levenberg-marquadrt algorithm.

\section{Research methods}

In order to find the best model to predict wind speed on a short time scale, we collected meteorological data from the city of Laayoune [8-9], taken every day (Daily) throughout the three years 2017-2018-2019. The parameters are wind speed (WS) in $\mathrm{m} / \mathrm{s}$, wind direction (DIR) in deg, temperature (TMP) in ${ }^{\circ} \mathrm{C}$, air pressure (ATM) in $\mathrm{hPa}$ and humidity $(\mathrm{H})$ in $\%$. We prepared the data in Excel and then trained it in Matlab.

\subsection{Neural Network}

A neural network is part of artificial intelligence [10] and in particularly, in deep learning sphere, which is a science, inspired by brain activities. In this study, we have chosen the NARX neural network, a feedback neural network, i.e. recurrent artificial neurons network [11]. We worked with both architectures, NARX parallel or closed loop, and NARX serial-parallel or open loop, we kept the same architecture for both networks, a threelayer architecture, an input layer with 5 inputs, an intermediate layer and an output layer containing a single output. Any artificial neural network is a multilayer perceptron (in all cases of learning, either supervised learning or unsupervised learning).

\subsubsection{Multilayers perceptron (MLP)}

The multilayer perceptron is a combination of linear separators which allows producing an overall non-linear separator. MLP is a neural network of a classical hierarchy having inputs or a single input, weights and Bias corresponding to the inputs, a linear summation function, an activation function and one or more outputs, the network is defined by the following relation:

$$
y=a\left(\sum_{n=1}^{p} w_{j} x_{j}+w_{0}\right)
$$

\footnotetext{
* Corresponding author: yousra.ame@gmail.com
} 
There are several types of classical neural networks such as Adaline (Adaline (Adaptive Linear Neuron), Madaline (Multi-Adaline) also RBF neural network (Radial Basis Function), the latter replaces the sigmoid activation function by radial basis function [12].

\subsubsection{NARX Close Loop}

NARX is a recurrent, i.e. cyclic or loop, neural network. NARX is based on the ARX time series. One can have open-loop NARX (NARX-SP) and closed-loop NARX (NARX-P) [13]. NARX-P is characterized by the feedback of past estimated values from the actual output to the input (an early feedback), and is a real-time configuration. The function that represents the operation of the NARX model in closed loop mode is:

$$
\hat{y}(t+1)=f\left(\hat{y}_{p}(t), u(t)\right)
$$

\subsubsection{NARX Open Loop}

The NARX open-loop network generally has two inputs, the first input based on the inputs $\mathrm{x}(\mathrm{t})$ or $\mathrm{u}(\mathrm{t})$ which is the sign of the inputs, and the second input presents the actual output of the network $y(t)$, it is used as it is, instead of returning the estimated output. While the regressor of the output is formed only by real values of the target series, i.e. the output of the network $y(t+1)$ so that the (SP) mode is used for learning between the target variables and the components which are the input series.

$$
\hat{y}(t+1)=f\left(y_{s p}(t), u(t)\right)
$$

Some studies have used NARX for forecasting wind speed, hourly solar radiation, forecasting network traffic, pollution, etc.

Annalisa Di Piazza et al used NARX to perform the hourly forecast of solar irradiation and wind speed, considering temperature as an exogenous variable. NARX was based on two techniques, the optimization technique based on a genetic algorithm (GA) and method that determine the optimal network architecture by pruning (optimal brain surgeon (OBS) strategy) [14].

Hong Thom Pham et al, chose to work with a highpowered hybrid NARX-ARMA model in order to predict the long-term condition of a machine. This forecast was based on vibration data. NARX is used to predict the deterministic component and ARMA to predict the error component [15].

As the NARX model is a type of dynamic neural network. Ines Sansa et al used a simulation to prove NARX's predictive effectiveness against static network models [16].

\subsection{Input variables}

The table below shows the correlation between the variables used. The correlation coefficient is used to select the important parameters. The table shows that all the weather parameters mentioned are to be used because all the correlation coefficients are low.

Table 1. Presentation of correlation coefficients between variables.

\begin{tabular}{|c|c|c|c|c|}
\hline Variables & WS & Pre & Temp & Hum \\
\hline Wind Speed & 1 & $-\mathbf{0 , 1 5 8}$ & $\mathbf{0 , 0 6 3}$ & $\mathbf{- 0 , 0 9 4}$ \\
\hline Pressure & & 1 & $-\mathbf{0 , 4 2 0}$ & $-\mathbf{0 , 1 5 3}$ \\
\hline Temperature & & & 1 & $-\mathbf{0 , 1 4 8}$ \\
\hline Humidity & & & & 1 \\
\hline
\end{tabular}

\section{Results and discussion}

The objective of the study is to find the most suitable network among NARX-P and NARX-SP for the daily prediction of the wind speed of Laayoune city. We optimized the learning in both networks by the Levenbrg-Marquardt optimization algorithm [17-19].

LM combines the advantageous techniques of the Gauss-Newton and gradient descent methods, is the derivative of the Netwon method, a second-order optimization technique, and used in the case of a continuous and differentiable function in a specific interval, it is performed to minimize functions that have sums of squares of non-linear functions.

The Levenberg-Marquardt algorithm is expressed as:

$$
w_{k+1}=w_{k}-\left(J_{k}^{T} J_{k}+\mu I\right)^{-1} J_{k} e_{k}
$$

Table 2. Training function and associated algorithms

\begin{tabular}{|c|c|}
\hline $\begin{array}{c}\text { Méthodes } \\
\text { d'entrainement }\end{array}$ & $\begin{array}{c}\text { Algorithmes d'Optimisation } \\
\text { associé }\end{array}$ \\
\hline $\begin{array}{c}\text { Levenberg-Marquardt } \\
(\mathrm{LM})\end{array}$ & $\begin{array}{c}\text { Gauss-Newton method + Gradient } \\
\text { Descent method }\end{array}$ \\
\hline
\end{tabular}

First of all, choosing the right training function helps to predict the wind speed very efficiently, i.e. to learn the non-linear behavior perfectly. Secondly, The correct choice of parameters such as the number of inputs, the number of neurons, and the correct activation function in the hidden layer also plays a primary role in the correct adjustment of the weights and subsequently in obtaining a or several optimal wind prediction models. 
We have chosen the same architecture for the two neural networks; we have five inputs (t-1): WS (t-1), DIR (t-1), ATM (t-1), H (t-1), TMP (t-1) in the input layer, a hidden layer with 10 neurons and hyperbolic tangent activation function, and only one output in the output layer with linear activation function.

\subsection{NARX Closed loop (or parallel) (18-10-1)}

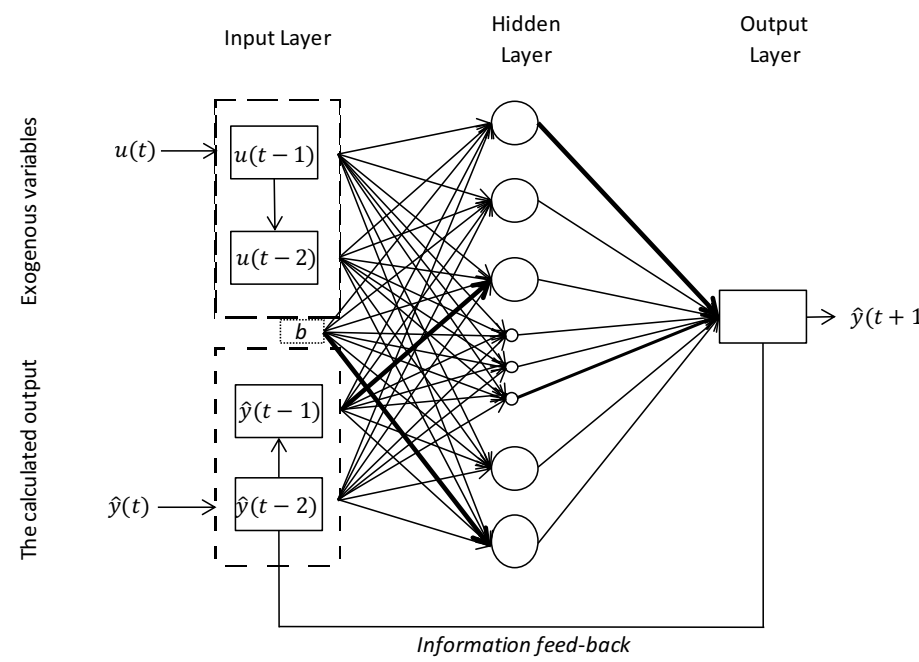

Fig. 2. NARX-P Representation $(18,10,1)$

The NARX-P model, with 18 inputs $(5 \times 3$ of the exogenous inputs $)$ and $(1 \times 3$ of the inputs from the output), the number of inputs is under the condition of the chosen delays, [1:2], represents the exogenous series (u) at present, time (t-1) and past (t-2), as well as for the input from the output $\hat{y}$ is at times $(\mathrm{t}),(\mathrm{t}-1),(\mathrm{t}-2)$, a hidden layer of 10 neurons connected by the hyperbolic tangent function, and an output activated by the linear function.

With:

$u(t)=\left(x_{1}(t), \ldots, x_{5}(t), x_{1}(x-1), \ldots, x_{5}(x-\right.$ 1), $\left.x_{1}(x-2), \ldots, x_{5}(x-2)\right)$

and

$$
\hat{y}(t)=(\hat{y}(t), \hat{y}(t-1), \hat{y}(t-2))
$$

Then:

$\hat{y}(t+1)=f(\hat{y}(t), \hat{y}(t-1), \hat{y}(t-2), u(t), u(t-$ 1), $u(t-2))$

The input to the perceptron in general for NARX-P and NARX-SP is:

$$
y=s\left(W_{x}^{T} X+w_{y} y+b\right)
$$

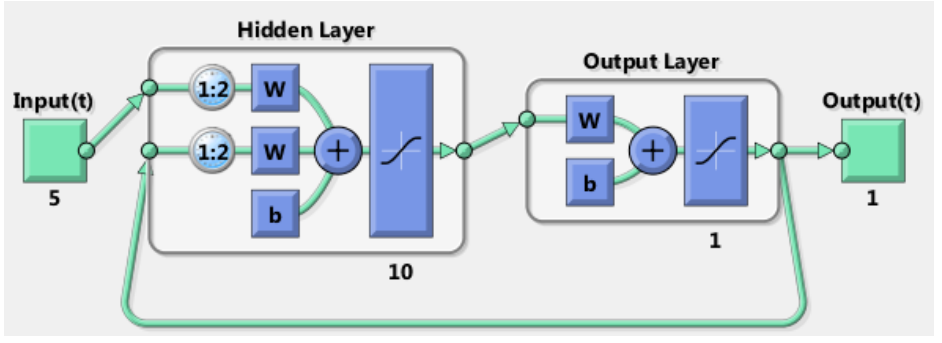

Fig. 3. NARX Neural Network - 10 Neurones - Tansig fonction activation

NARX close loop type, it has 10 hidden layer neurons, and a Tanh function (hyperbolic tangent), similar to the sigmoid function except that this first one produces results between -1 and 1 (centered in zero).

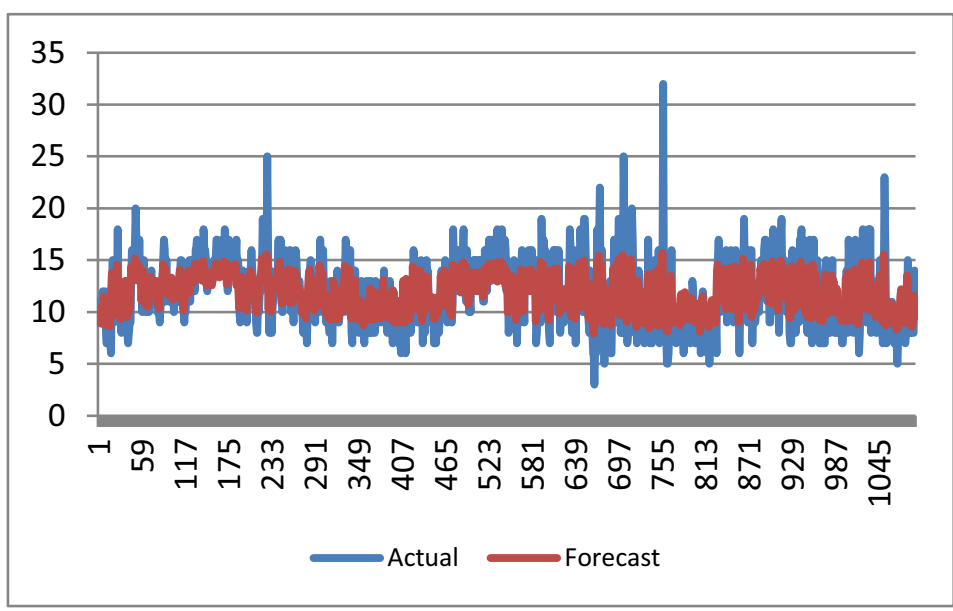

Fig. 4. The actual wind speed series and the series estimated by NARX-P

From Figure 4, it can be seen that the NARX-P network was unable to correctly predict the wind speed variables.

\subsection{NARX Open loop (or series-parallel) (18-10- 1)}

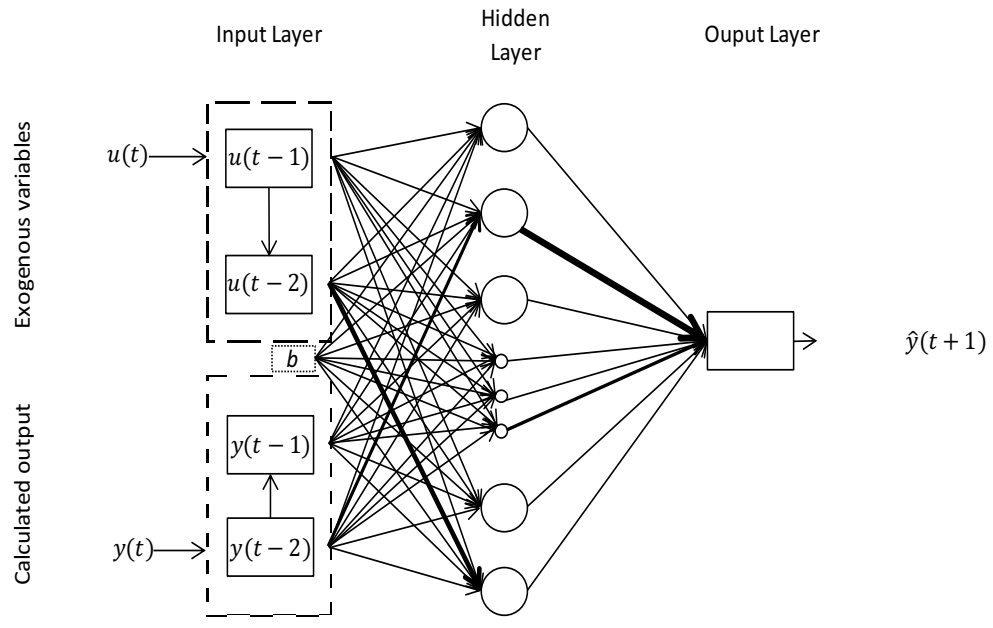

Fig. 5. NARX-SP Representation $(18,10,1)$ 
NARX-SP keeps the same structure as NARX-P, the objective is to compare the two models with the same parameters.

With:

$u(t)=\left(x_{1}(t), \ldots, x_{5}(t), x_{1}(x-1), \ldots, x_{5}(x-\right.$ 1), $\left.x_{1}(x-2), \ldots, x_{5}(x-2)\right)$

And

$$
y(t)=(y(t), y(t-1), y(t-2))
$$

Then:

$\hat{y}(t+1)=f(y(t), y(t-1), y(t-2), u(t), u(t-$ 1), $u(t-2))$

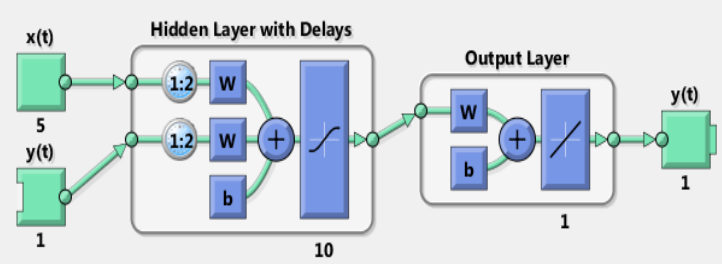

Fig. 6. NARX -SP Neural Network - 10 Neurones - Tansig fonction activation

NARX open loop type, it has 10 hidden layer neurons, and a Tanh function (hyperbolic tangent).

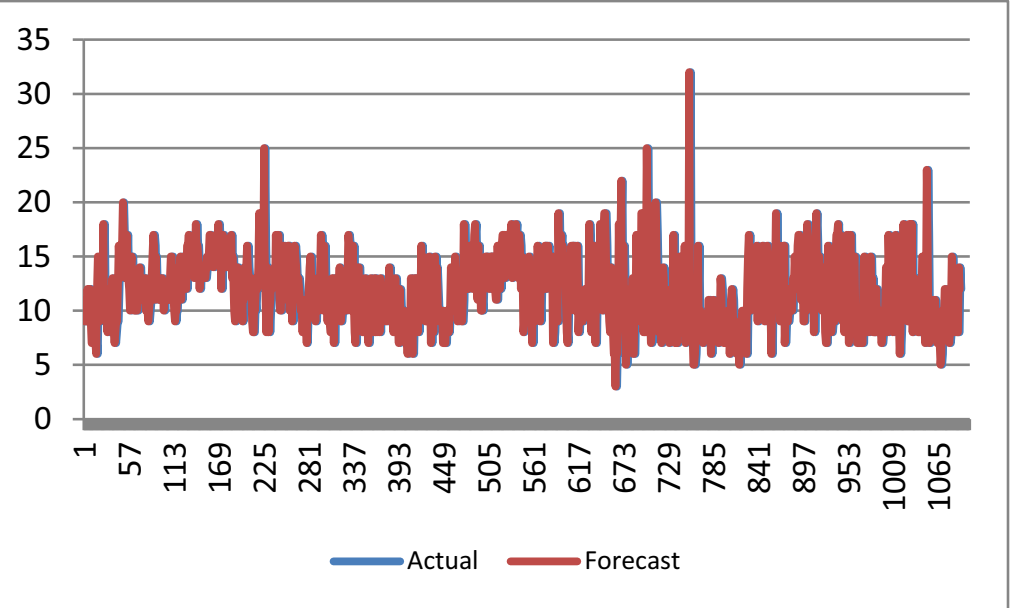

Fig. 7. The actual wind speed series and the series estimated by NARX-SP

The NARX-SP model was able to learn and subsequently predict the wind speed series perfectly. The network architecture was chosen after several trials, we changed the number of hidden layers as well as the number of neurons in the hidden layer, but the chosen architecture presented the best results among others.

In this study, we should have predicted quantitative variables, i.e. it is a multiple linear regression problem ( 5 inputs and a single output). For this regression analysis, we used four types of forecast error measures, as shown in the table below. RMSE [20].

RMSE (Root Mean Square Error) $=\sqrt{\frac{1}{n}} \sum_{1}^{n}(O-d)^{2}$

\begin{tabular}{|c|c|c|}
\hline & NARX-SP & NARX-P \\
\hline RMSE & 0,35557 & 2,6309 \\
\hline
\end{tabular}

\section{Conclusion}

This study attempts to find the most appropriate network for predicting the maximum wind speed of the city of Laayoune. We chose two different architectures of the NARX recurrent neural network, closed loop and open loop, trained by the Levenberg-Marquardt algorithm. NARX-SP showed good similarity, and the estimated variables were very close to the real variables.

\section{References}

1. A. Alhamwi, D. Kleinhans, S. Weitemeyer, T. Wogt, Moroccan National Energy Strategy reviewed from a meteorological perspective, Energy Strategy Reviewed 6, p. 39-47 (2015)

2. M. Azeroual, A. El Makrini, H. El Moussaoui, H. El Markhi, Renewable Energy Potential and Available capacity for wind and solar Power in Morocco Towards 2030, Journal of Engineering Science and Technology Review 11(1), p. 189-198 (2018)

3. A. Nouri, M. Ait Bahram, E. Elwanaki, M. Enzili, Moroccan wind farm potential feasibility. Case Study, Energy conversion and Management 112, p. 39-51 (2016)

4. A. Allouhi, Energetic, exergetic, economic and environmental (4E) assessment process of wind power generation, Journal of Cleaner Production 235, p.123-137 (2019)

5. Y. Amellas, O. El Bakkali, A. Djebli, A. Echchelh, Short-term wind speed prediction based on MLP and NARX networks models, Indonesian Journal of Electrical Engineering and Computer Science, Vol 18, N¹, p.150-157 (2020)

6. R. Sharma, D. Singh, A review of wind Power and Wind Speed Forecasting, Journal of Engineering Research and Application 8, Issue 7( Part 3), p.0109 (2018)

7. E. Erdem, J. Shi, ARMA based approaches for forecasting the tuple of wind speed and direction, Applied Energy 88, p. 1405-1414 (2011)

8. Y. El Khchine, M. Sriti, N. Eddine El Kadri Elyamani, Evaluation of wind energy potential and trends in Morocco, Heliyon 5, e01830 (2019)

9. H. Bidaoui, I. El Abbassi, A. El bouardi, A. Darcherif, Wind Speed Data Analysis Using Weibull and Rayleigh Distribution Function, Case Study: Five cites Northern Morocco, Procedia Manufacturing, Vol 23, p.786-793 (2019) 
10. A. El Ibrahimi, A. Baali, Application of several Artificial Intelligence Models for forecasting Meteorological Drought Using the standardized precipitations Index in the Saiss Plain (Northern Morocco), International Journal of Intelligent Engineering ans Systems, Vol 11, N¹, p.267-275 (2018)

11. G. Gelly, Réseaux de neurones récurrents pour le traitement automatique de la parole ( Thèse de Doctorat ) (2017)

12. G.W, H.J. Lu, Y.R. Chang, Y.D.Lee, An improved neural network-based approach for short-term wind speed and power forecast, Renewable Energy 105, p.301-311 (2017)

13. A. A. Hussein, A Derivation and comparison of open loop and closed loop Neural Network Battery, State-of-Charge Estimators, Energy Procedia 75, p.1856-1861 (2015)

14. A. Di Piazza, M. Carmela Di Piazza, G. Vitale, Solar and Wind forecasting by NARX Neural Networks, Renewable Energy and Environmental Sustainable 1, 39 (2016)

15. T. Van Tung, P, H.Thom, Yang, Bo $\neg$ Suk ,An improved hybrid of nonlinear auto-regressive with exogenous input and autoregressive moving average for long $\neg$ term machine state forecasting based on vibration signal. Expert Systems With Applications, 37 (4). p. 3310- -3317. ISSN 0957$\neg 4174$ (2010)

16. I. Sansa, N. Mrabet Bellaaj, Solar Radiation using NARX model, Advanced Applications for Artificial Neural Network, Chapter 13 (2018)

17. S. Mammadi, Financial time series prediction using artificial neural network based on LevenbergMarquardt algorithm, 9th International International Conference on Theory and Application of Soft Computing, Computing with words and Perception, ICSCCW, Procedia Computer Science 120, 602-607 (2017)

18. M. Hock Fun, M.T. Hagan, Levenberg-Marquardt Training for Modular Networks, Proceedings of International Conference on Neural Networks (ICNN'96) (1996).

19. Y.Amellas, A.Echchelh, Levenberg-Marquardt Training Function using, MLP, RNN and Elman Network to Optimize Hourly Forecasting in Tetouan City (Northern Morocco), Journal of Engineering Science and Technology Review 13 (1) 67-71 (2020).

20. M.V. Shcherbakov, A.Brebels, N.L. Shcherbakova, A.P. Tyukov, T.A. Janosky, V.A.Kamaev, Survey of Forecast Error Measures, World Applied Sciences Journal 24 (Information Technologies in Modern Industry, Education and Society ), p. 171176 (2013) 